\title{
Additions to the Pantepui pollen flora (Venezuelan Guayana): the Maguire Collection
}

\author{
C. LÓPEZ-MARTÍNEZ ${ }^{1}$, A. LARA ${ }^{1}$, V. RULL ${ }^{1}$, L. CAMPBELL ${ }^{2} \&$ S. NOGUÉ 3 \\ ${ }^{1}$ Palynology and Paleoecology Lab, Institut Botànic de Barcelona (CSIC-ICUB), Psg. del Migdia s/n., 08038 Barcelona, Spain \\ ${ }^{2}$ Plant Research Lab, The New York Botanical Garden, Bronx, NY 10458, USA \\ ${ }^{3}$ Long Term Ecology Lab, Oxford University Centre for the Environment, South Parks Road, Oxford OX1 3QY, UK
}

Author for correspondence: V. Rull (vrull@ibb.csic.es)

Received 3 September 2010; Accepted 15 November 2010

\begin{abstract}
This work is a pollen-morphological study of various plant species from Pantepui (Venezuelan Guayana), a region with high biodiversity and endemism, where global warming is expected to have a high impact. The study consists of a series of morphological descriptions of selected taxa from the Maguire pollen reference slide collection of The New York Botanical Garden (NYBG). The collection was initiated under the supervision of Senior Curator Bassett Maguire to advance systematic, palynological, and medical studies; today it has become also useful for other disciplines such as paleoecology, paleoclimatology or forensic studies. The aim of this pollen-morphological study is to enhance the database of pollen descriptions and illustrations for identification purposes, to be used in the ongoing paleoecological reconstructions and, eventually, in other types of studies using pollen, particularly from the Guayanan tepui summits.
\end{abstract}

Key words: Guayana; Pollen morphology; Taxonomy; Tepuis; Venezuela.

\section{Resumen}

Adiciones a la flora polínica de Pantepui (Guayana venezolana): la colección Maguire.- Este trabajo es un estudio sobre la morfología polínica de varias especies de plantas de Pantepui (Guayana venezolana), una región con elevada biodiversidad y un alto grado de endemismo, donde se espera un fuerte impacto del calentamiento global. El estudio consiste en una serie de descripciones morfológicas de taxones pertenecientes a la colección Maguire de láminas de referencia del Jardín Botánico de Nueva York (NYBG). La colección fue iniciada bajo la dirección del Curador Jefe Basset Maguire con el objeto de avanzar en estudios de sistemática, palinología y medicina; aunque actualmente también resulta útil en otras disciplinas, como por ejemplo la paleoecología, la paleoclimatología o los estudios forenses. El objetivo de este estudio de morfología polínica es aumentar la base de datos de descripciones e ilustraciones polínicas con propósitos de identificación taxonómica, para ser utilizada en reconstrucciones paleoecológicas y otro tipo de estudios actualmente en desarrollo, en particular en las cimas de los tepuis de Guayana.

Palabras clave: Guayana; Morfología polínica; Taxonomía; Tepuis; Venezuela. 


\section{INTRODUCTION}

This paper is part of a series devoted to the pollenmorphological study of vascular plant species from the Venezuelan Guayana (Fig. 1), particularly from the characteristic sandstone tabular mountains (tepuis), initiated by Salgado-Labouriau \& Villar (1992) and continued by Rull (2003). The biodiversity and endemism of this region are striking, but the origin and evolution of such exceptional biotic features has been object of intense debate, which is still ongoing (Rull, 2010). On the other hand, the tepuian flora is expected to be seriously affected by relatively rapid biodiversity loss under ongoing global warming. Hence, paleoecological and paleoclimatic studies on this region can provide useful information about biogeographical and evolutionary processes as well as the effects of climate change on the tepuian biota.

Besides the papers mentioned above, which are monographic for the pollen of the Guayana region, the available literature dealing with this subject is scarce and organized taxonomically. Rapateaceae and Asteraceae are the most thoroughly studied families in this sense, although the knowledge of Asteraceae is restricted to some genera of Mutisioideae (Carlquist, 1961; Tellería, 2008; Funk et al., 2009; Ubiergo et al., 2009). Accordingly, the aim of this study, as well as the previously mentioned works, is to enhance the database of pollen descriptions and illustrations for identification purposes, to be used in the ongoing pollen-based paleoecological reconstructions (Rull et al., 2010) and, eventually, in other types of studies using pollen. This work is based on the Maguire pollen reference slide collection, initiated in the forties of the past century by Senior Curator B. Maguire of the New York Botanical Garden (NYBG) to make progress in biological research. Most genera and/or species studied here are endemic to the Guayana region or even to the Pantepui province or a single tepui.

\section{Study area: Pantepui}

In the local indigenous language (Pemón), "tepui" means "stone bud", and is the name for the characteristic sandstone/quartzite tabular mountains of Guayana (Fig. 2). They vary in altitude and extension, ranging from around 1100 to $3000 \mathrm{~m}$ (typically 2000-2600 m) of maximum elevation, and with a surface of $<1$ to $>1000 \mathrm{~km}^{2}$ (typically $200-500 \mathrm{~km}^{2}$ ) (Huber, 1995a). The tepui summits are more or less flat and hold unique and specialized life forms and communities, as well as a high diversity and an amazing degree of endemism (Rull, 2009). This biotic singularity led to the creation of the Pantepui biogeographical province, which embraces all the tepui summits above $1500 \mathrm{~m}$ elevation (Huber, 1988, 1994). Vascular plants are the better known organisms of Pantepui, with almost 2500 species, of which $>60 \%$ are endemic to the Guayana region and $>40 \%$ are endemic to Pantepui, including $25 \%$ species endemic to a single tepui (Berry \& Riina, 2005). Similarly, most tepuian vegetation types are unparalleled worldwide (Huber, 2005). Pantepui is mostly pristine due to its remoteness and inaccessibility; only a few of the tepui summits can be reached by foot after several days of walking and climbing. Indigenous people living in the surrounding lowlands and midlands do not visit the tepui summits because they consider them the home of gods and access is not allowed. On the other hand, there is no potential for commercial profit through activities such as mining, agriculture, cattle raising, etc. (Gorzula \& Huber, 1992; Huber, 1995b). Tourism and biopiracy are restricted by severe regulations that, unfortunately, also hinder scientific research (Rull \& Vegas-Vilarrúbia, 2008; Rull et al., 2008a). The main threat for the Pantepui biota is global warming that, according to recent estimates, would determine significant biodiversity losses by upward migration and the corresponding habitat loss and fragmentation for many endemic species (Rull \& Vegas-Vilarrúbia, 2006; Rull et al., 2008b; Nogué et al., 2009).

\section{MATERIAL AND METHODS}

Slides for this study are part of the Maguire pollen reference slide collection preserved in The New York Botanical Garden (NYBG; see http://www.nybg.org/). The collection comprises 3650 preparations of modern pollen representing 153 vascular plant families, of which 11 are non-seed plants. Some preparations include duplicate slides. Most vouchers are deposited in the Steere Herbarium of NYBG and can be viewed on-line (http://www.nybg.org/). The collection was initiated under the supervision of Senior Curator Bassett Maguire (1904-1991) to advance systematic, palynological, and medical studies. Research utilizing 


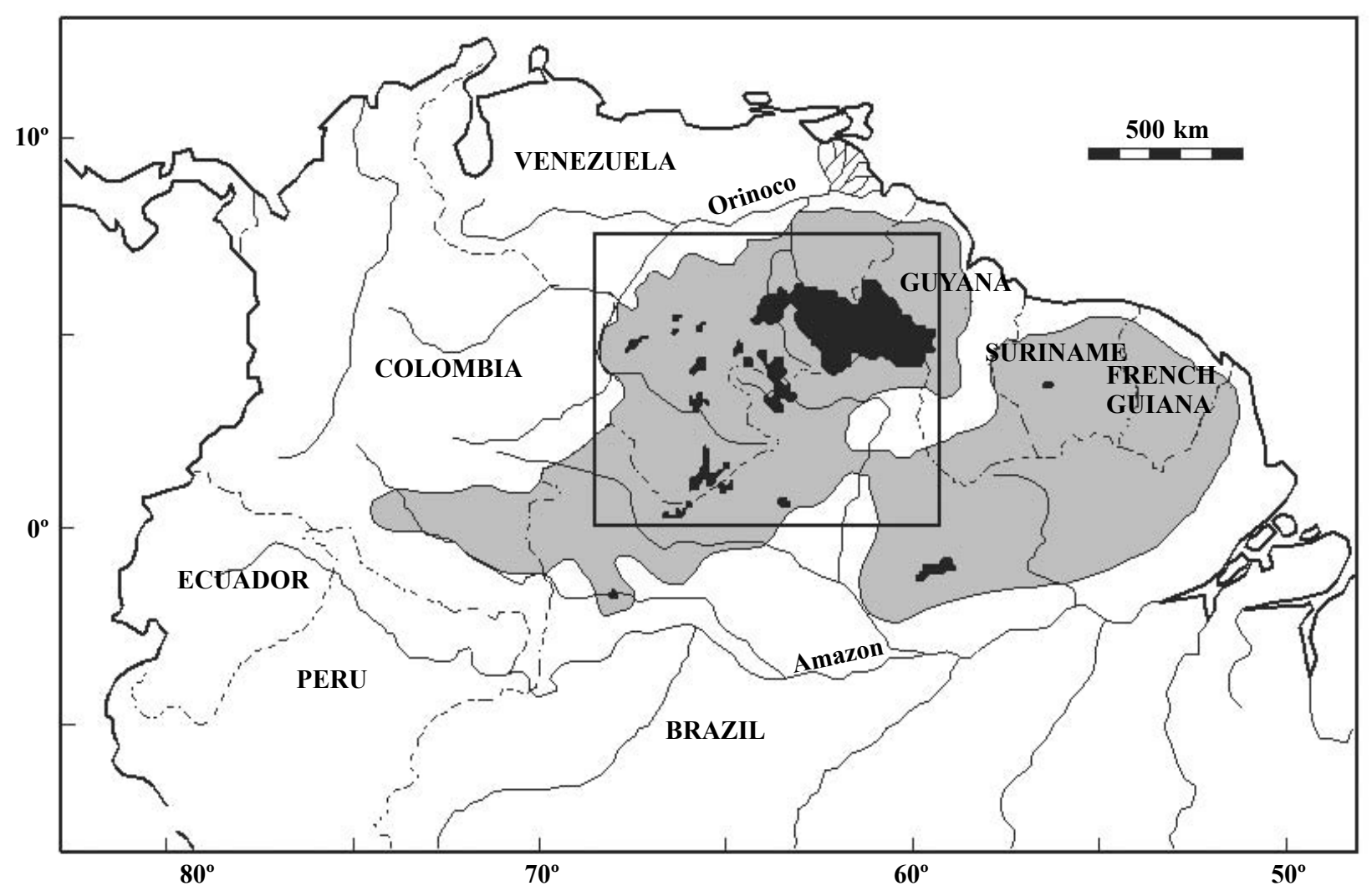

Figure 1. Map of northern South America, showing the approximate extension of the Guayana Shield (in grey) and the Precambrian sandstones and quartzites of the Roraima group (in black), on which the tepuis are modelled (Briceño \& Schubert, 1990). The region under study (the Venezuelan Guayana) is indicated by a box.

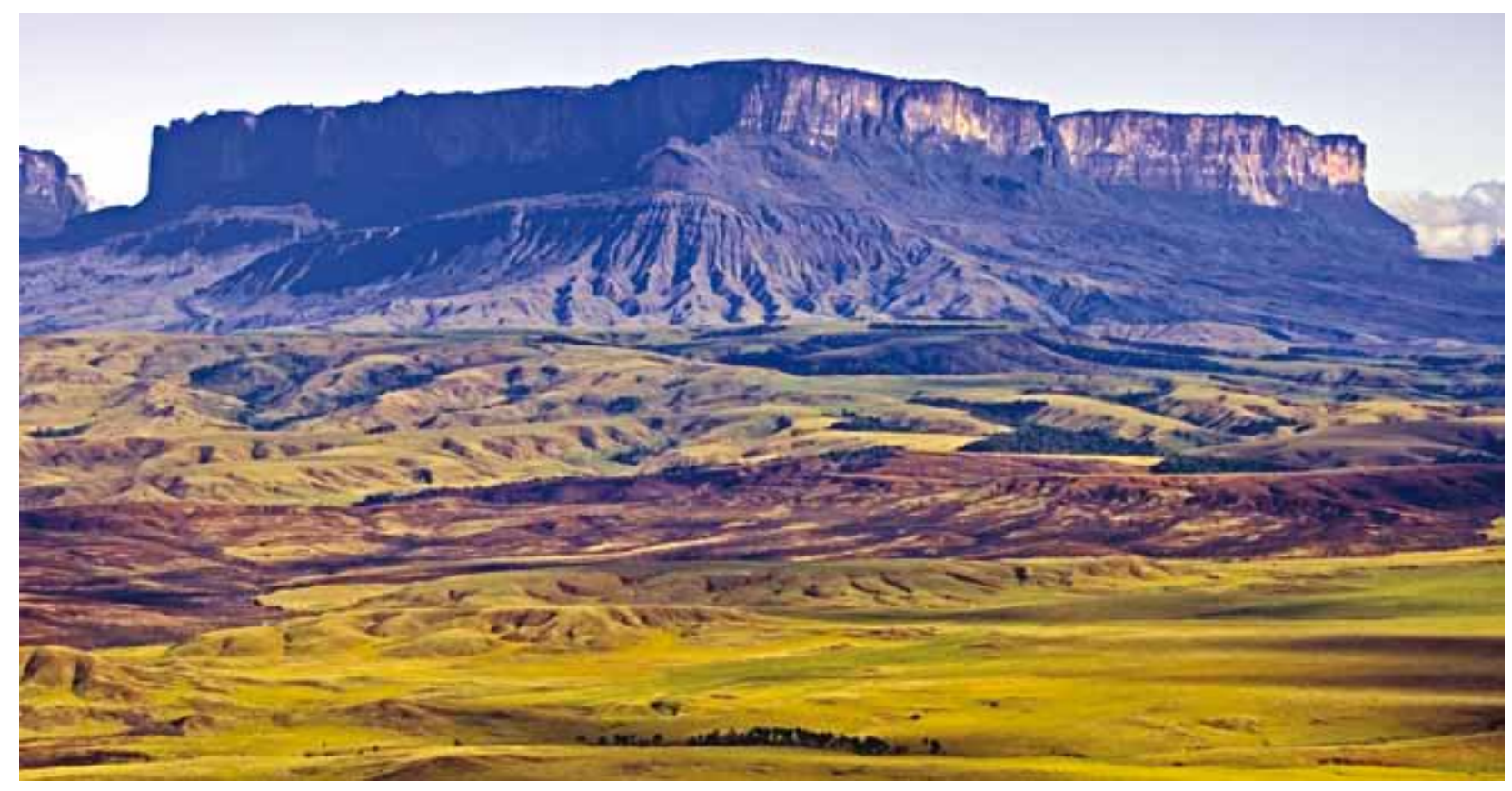

Figure 2. Example of a tepui: picture of the Roraima tepui [Photograph by V. Rull]. 
these specimens will undoubtedly surpass the original objectives by serving fields as for example paleoecology, climate change or forensic studies, among others. The breadth of the collection is international in scope with the greatest geographic representation in tropical America, particularly the Guayana region of northern South America. Systematic strengths of the collection reflect Maguire's interest in plant groups with high species diversity or endemism in the Guayana (e.g., Clusiaceae, Gentianaceae, Rapateaceae, and Velloziaceae). Samples for the Maguire collection were mostly obtained from dried or fluid preserved collections housed at NYBG, but also include dried or fresh material as well as prepared slides from other institutions. Pollen grains were chemically treated with $\mathrm{KOH}$, acetolysis, a combination of the two, or were untreated, and mounted in glycerin jelly. The slides are stored in vertical sleeves in a steel cabinet (see Howard \& Boom, 1990, Fig. 11).

Measurements for this work have been taken in all the available well-preserved grains; the number of grains used in each case is indicated after the measurements $(\mathrm{n}=\mathrm{x})$. Measurements were taken using the photographic software DPXViewer 1.14.9 and a DeltaPix InfinityX camera. A number of species could not have been described because of the absence of suitable pollen grains due to bad preservation. These taxa are listed in the appendix for reference. Taxa are ordered alphabetically by families, following the classification of the Flora of the Venezuelan Guayana (Steyermark et al., 1995-2005). For each plant species there is a morphological description accompanied by some photographs of the pollen grains found in the slide. The descriptions follow the Rull (2003) style for consistency, and use the standard nomenclature system of Punt et al. (2007). The title of every description summarizes the genus and species, the species authors, the pollen catalogue number, the collector, the collection location, as well as other information present on the label of the slide. Within the description, colpi and ora sizes are expressed as length $\mathrm{x}$ width (regarding the direction of the polar axis, in equatorial view), and grain size, as length of the polar axis $\mathrm{x}$ equatorial diameter. Also, the more recently accepted name of the species is noted where necessary, as well as the literature where pollen of other species of the same genera from the Venezuelan Guayana are described, if any. The following abbreviations have been used in the text and descriptions: PV, polar view; EV, equatorial view; $\mathrm{P} / \mathrm{E}$, ratio of the length of the polar axis $(\mathrm{P})$ to the equatorial diameter $(\mathrm{E})$ (this ratio is the basis of a system of shape classes, Punt et al., 2007). Finally, the photographs are ordered by pollen morphology (monads, tetrads and polyads, and the number and type of apertures). The scale bar in the plates is always $10 \mu \mathrm{m}$ long, except for the images of Moronobea jenmanii Engl. in Mart. var. fanshawei Maguire and Irlbachia cardonae (Gleason) Maguire, where it is $20 \mu \mathrm{m}$ long.

\section{POLLEN DESCRIPTIONS}

\section{Asteraceae}

\section{Glossarion rhodanthum Maguire \& Wurdack (Plate 1: 6-9) \\ (NY 2584; B. Maguire et al. 37126; Neblina)}

Monads. Isopolar, subprolate $(\mathrm{P} / \mathrm{E}=1.27), \mathrm{amb}$ circular slightly lobate. Tricolporate, zonoaperturate. Ectocolpi long, sharp ends, fusiform $(74.2 \times 4.2 \mu \mathrm{m})$, marginate. Ora fusiform, lalongate $(7.4 \times 25.8 \mu \mathrm{m})$. Exine $8.8 \mu \mathrm{m}$ thick (excluding spines), crescentic (in polar view, very thick exine in the medium of the intercolpium, gradually thinning towards the colpi), caveate, tectate, echinate, supratectal spines triangular (ca. $3 \mu \mathrm{m}$ long and $3 \mu \mathrm{m}$ wide at the base) with slightly rounded ends. Collumellae gradually more dense and/or smaller near the margo. Sexine thicker than nexine. Grain size (excluding spines): 89.8-102.8 x 71.4-80.5 $\mu \mathrm{m}, \mathrm{n}=10$.

\section{Gongylolepis benthamiana R. Schomb. (Plate 1: 10-13)}

\section{(NY 2586; T. Koyama \& G. Agostini 7366; Venezuela)}

Monads. Isopolar, subprolate to prolate $(\mathrm{P} / \mathrm{E}=1.33)$, amb circular slightly lobate. Tricolporate, zonoaperturate. Ectocolpi long, rounded ends, elliptic $(83.2 \times 4.2 \mu \mathrm{m})$, margo thick $(4.0 \mu \mathrm{m})$, psilate. Ora elliptic, lolongate $(19.9 \times 12.3 \mu \mathrm{m})$. Exine $9.3 \mu \mathrm{m}$ thick (excluding spines), caveate, tectate, echinate, supratectal spines triangular ( $c a$. $4 \mu \mathrm{m}$ long and ca. $5 \mu \mathrm{m}$ at the base) with slightly

Plate 1. 1-3, Kunhardtia rhodantha (Rapateaceae); 4-5, Barbacenia magalhaesii (Velloziaceae); 6-9, Glossarion rhodanthum (Asteraceae); 10-13 Gongylolepis benthamiana (Asteraceae). 

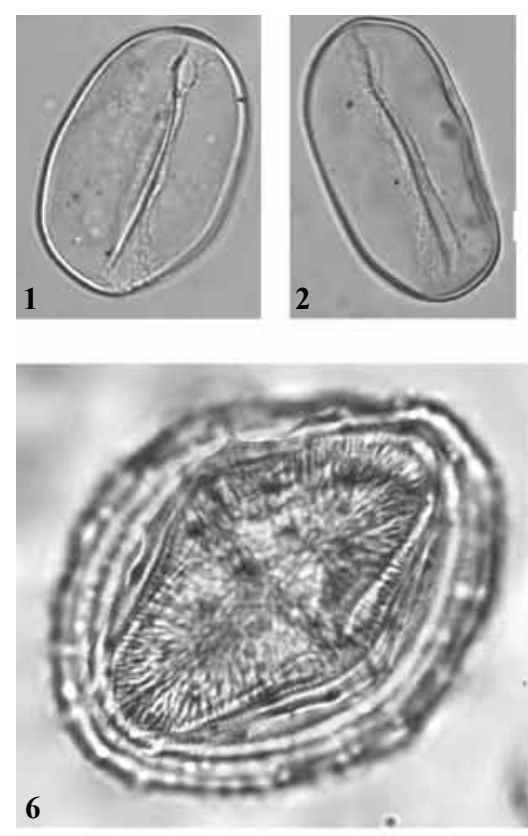
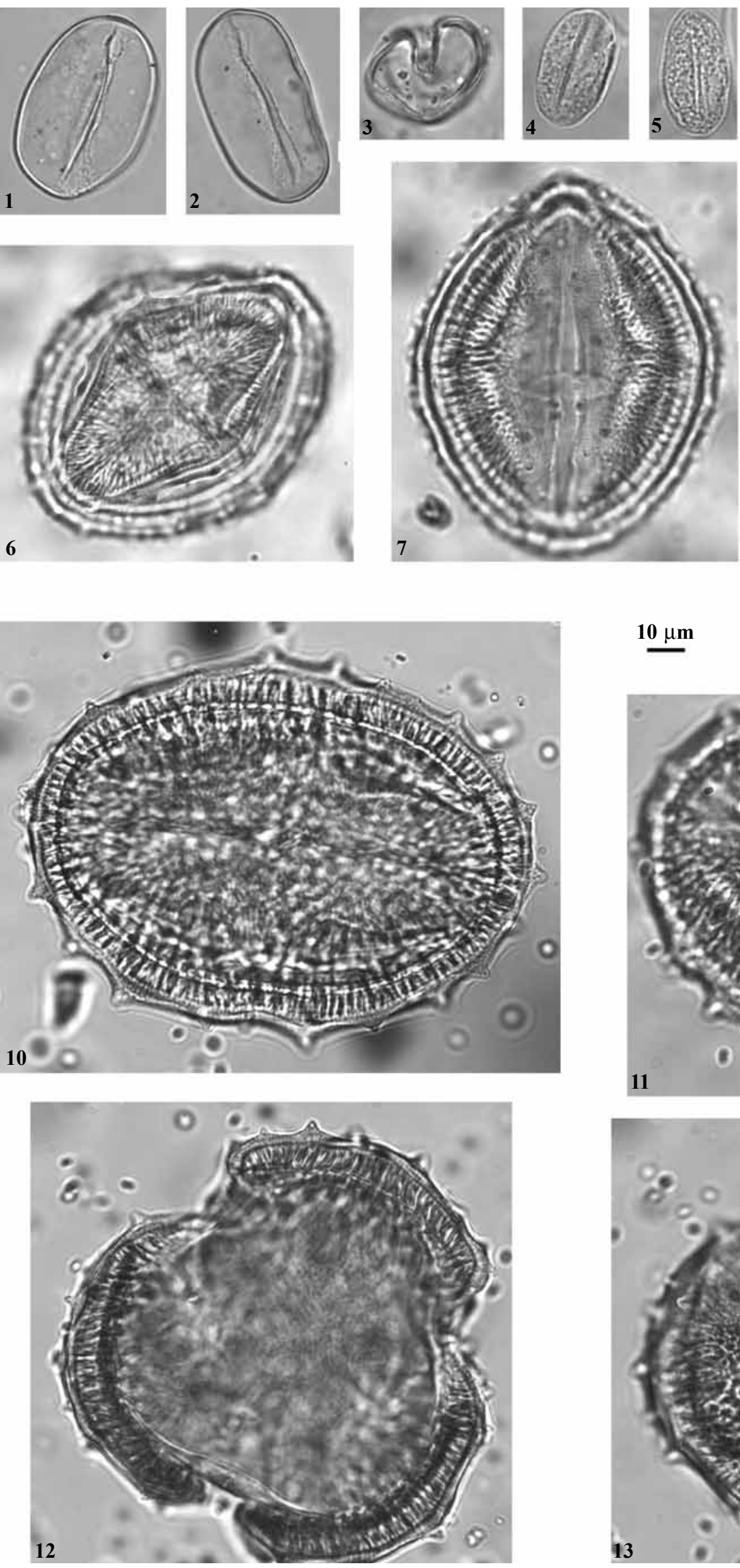
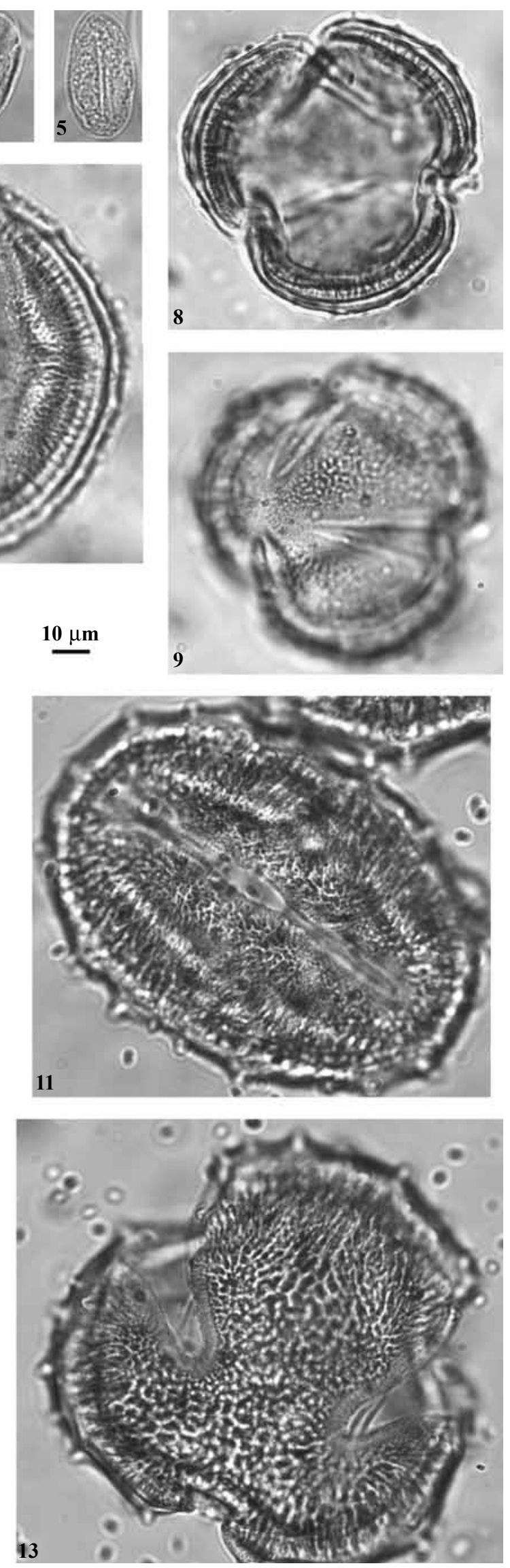
rounded ends. Sexine thicker than nexine. Grain size: 112.4-133.6 x 87.2-103.0 $\mu \mathrm{m}$ (excluding spines), $\mathrm{n}=11$.

Stenopadus cucullatus Maguire (Plate 2: 14-17) (NY 2592; B. Maguire \& C. K. Maguire 35029; Venezuela)

Monads. Isopolar, prolate $(\mathrm{P} / \mathrm{E}=1.53)$, amb circular slightly lobate. Tricolporate, zonoaperturate. Ectocolpi long, rounded ends, elliptic $(74.8 \times 3.7 \mu \mathrm{m})$, slightly marginate. Ora fusiform, lalongate $(7.6 \times 23.6 \mu \mathrm{m})$. Exine $3.1 \mu \mathrm{m}$ thick, thicker at the poles $(4.8 \mu \mathrm{m})$, tectate, psilate (columellae visible through the tectum). Sexine thicker than nexine. Grain size: $91.5-101.5$ x 56.6-69.9 $\mu \mathrm{m}, \mathrm{n}=11$.

Comments: Stenopadus sp. described in Rull (2003).

\section{Bonnetiaceae}

Bonnetia celiae Maguire (Plate 2: 18-21)

(NY 957; B. Maguire 35327; Venezuela)

Monads. Isopolar, subprolate $(\mathrm{P} / \mathrm{E}=1.31)$, amb sub-triangular, angulaperturate. Tricolporate, zonoaperturate. Ectocolpi long, sharp ends, fusiform $(50.8 \times 9.7 \mu \mathrm{m})$, marginate. Ora elliptic, lolongate $(12.8 \times 8.8 \mu \mathrm{m})$, annulus present. Exine $4.7 \mu \mathrm{m}$ thick, semitectate, perforate. Sexine thicker than nexine. Grain size: 54.0-74.4 x 43.1-57.3 $\mu \mathrm{m}, \mathrm{n}=9$.

Comments: Bonnetia lanceifolia Maguire, $B$. roraimae Oliver and $B$. sessilis Benth. are described in Salgado-Labouriau \& Villar (1992) and Rull (2003).

\section{Bonnetia roraimae Oliv. ex Thurn (Plate 2: 22-24) (104; Maguire 33421)}

Monads. Isopolar, oblate-spheroidal $(\mathrm{P} / \mathrm{E}=0.97)$, amb circular-triangular, angulaperturate. Tricolporate, zonoaperturate. Ectocolpi long, sharp ends, fusiform $(27.4 \mu \mathrm{m} \times 3.8 \mu \mathrm{m})$. Ora circular $(9.3 \mu \mathrm{m}$ diameter), sometimes operculate. Exine $2 \mu \mathrm{m}$ thick, semitectate, perforate. Sexine thicker than nexine. Grain size: $29.1-41.2 \times 27.3-43.0 \mu \mathrm{m}, \mathrm{n}=6$.

Comments: Bonnetia roraimae is described in Salgado-Labouriau \& Villar (1992) and Rull (2003).

\section{Clethraceae}

Clethra alnifolia L. (Plate 2: 25-28) (NY 3008; Martha's Vineyard, Mass, 18 Sept 1964; F. C. Mackoever)

Monads. Isopolar, prolate-spheroidal $(\mathrm{P} / \mathrm{E}=1.13)$, amb circular. Tricolporate, zonoaperturate. Ectocolpi long $(21.6 \mu \mathrm{m})$, shape difficult to distinguish. Ora lalongate, constricted, fastigium present. Exine $2.0 \mu \mathrm{m}$ thick, tectate, psilate. Sexine and nexine approximately of the same thickness. Grain size: 25.9-32.9 x 23.4-30.1 $\mu \mathrm{m}, \mathrm{n}=10$.

Comments: Clethra guianensis Klotzsch ex. Meisn. is described in Rull (2003).

\section{Clusiaceae}

Clusia cochlitheca Maguire (Plate 2: 29-30) (NY 3476; B. Maguire 36900)

Monads. Amb triangular, angulaperturate. Tricolporate, operculate (operculum not always present), zonoaperturate. Ectocolpi short $(12.3 \mu \mathrm{m})$, ora difficult to see. Exine $1.1 \mu \mathrm{m}$ thick, semitectate, perforate. Sexine and nexine indistinguishable for measuring. Grain size: 28.3-32.7 $\mu$ m, median in PV, $\mathrm{n}=5$.

Comments: Description based on grains in PV. Clusia melchiorii Gleason is described in Rull (2003).

\section{Clusia orthoneura Standl. (Plate 2: 31-34) (NY 3483; B. Maguire 62205)}

Monads. Isopolar, subprolate $(\mathrm{P} / \mathrm{E}=1.25), \mathrm{amb}$ triangular-circular, angulaperturate. Tricolporate, operculate (operculum not always present), zonoaperturate. Ectocolpi long $(29.5 \mu \mathrm{m})$, marginate, constricted in the equatorial region, sharp ends. Ora difficult to distinguish. Exine $1.8 \mu \mathrm{m}$ thick, semitectate, reticulate, heterobrochate (lumina slightly smaller near the colpi). Sexine slightly thiner than nexine. Grain size: $31.5-37.6 \times 25.3-$ $32.4 \mu \mathrm{m}, \mathrm{n}=20$.

Hypericum uliginosum H. B. K. (Plate 2: 35-36) (NY 753; E. P. Killip \& A. C. Smith 20487; Colombia)

Monads. Isopolar, subprolate $(\mathrm{P} / \mathrm{E}=1.22)$.

Plate 2. 14-17, Stenopadus cucullatus (Asteraceae); 18-21, Bonnetia celiae (Bonnetiaceae); 22-24, Bonnetia roraimae (Bonnetiaceae); 25-28, Clethra alnifolia (Clethraceae); 29-30, Clusia cochlitheca (Clusiaceae); 31-34 Clusia orthoneura (Clusiaceae); 35-36, Hypericum uliginosum (Clusiaceae); 37-40, Neotatea neblinae (Clusiaceae); 41-43, Tovomita weddelliana (Clusiaceae); 44-46, Tapeinostemon longiflorum (Gentianaceae). 

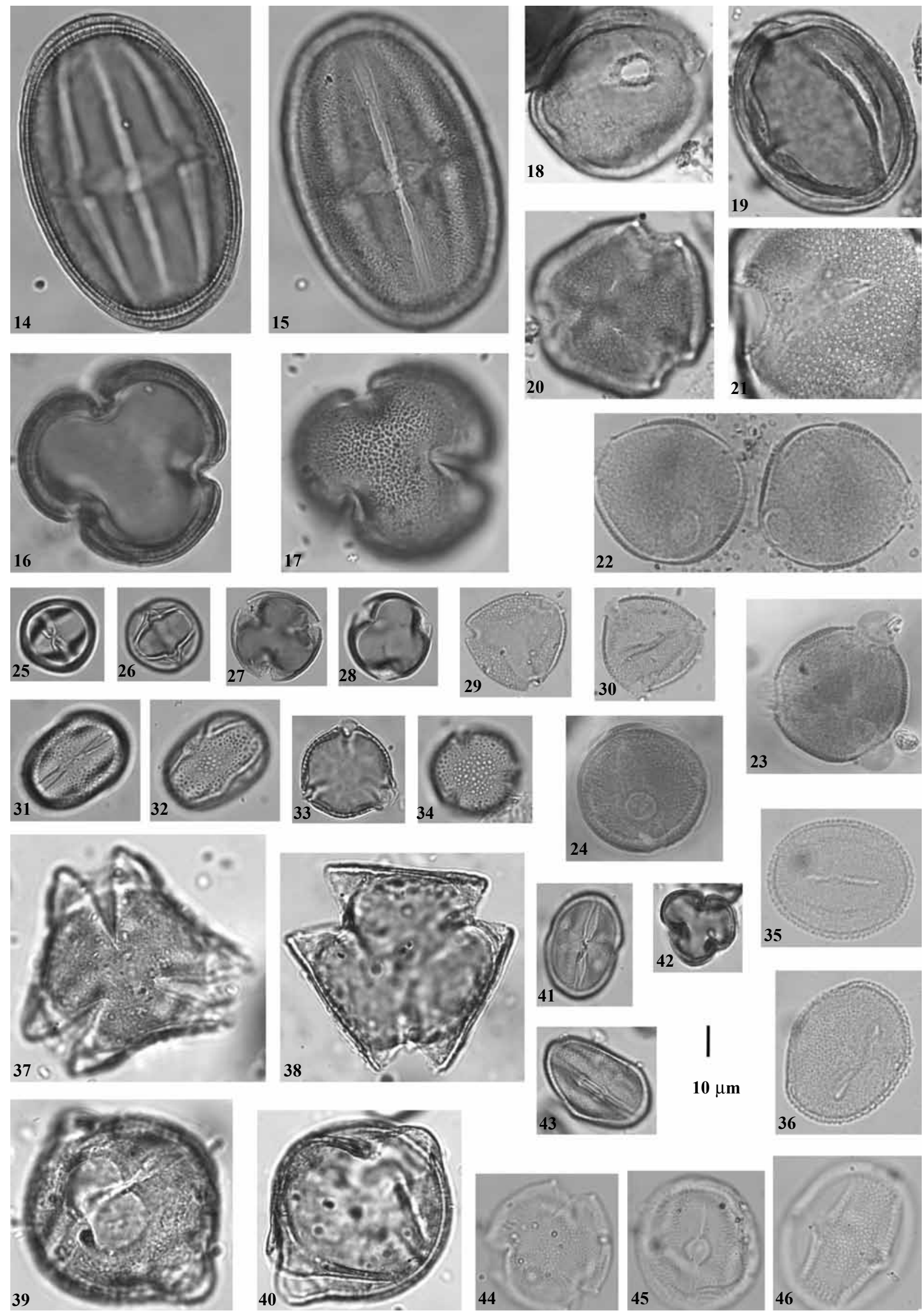

Collectanea Botanica vol. 29 (2010): 31-49, ISSN: 0010-0730, doi: 10.3989/collectbot.2010.v29.004 
Tricolporate, zonoaperturate. Ectocolpi long (27.5 $\mu \mathrm{m})$, rounded ends, marginate, ora difficult to see. Exine $2.3 \mu \mathrm{m}$ thick, semitectate, perforate. Sexine thicker than nexine. Grain size: 46.5-51.4 x 38.5$41.6 \mu \mathrm{m}, \mathrm{n}=4$.

\section{Moronobea jenmanii Engl. in Mart. var. fanshawei Maguire (Plate 3: 82-84) \\ (NY 2315; S. S. Tillett \& C. L. Tillett 44904; B. Guiana)}

Monads. Isopolar, subprolate $(\mathrm{P} / \mathrm{E}=1.18)$, amb circular-quadrangular angulaperturate. Tetracolporate (sometimes pentacolporate), zonoaperturate. Ectocolpi $94.3 \mu \mathrm{m}$ long and narrow. Ora difficult to see. Exine $4.7 \mu \mathrm{m}$ thick, psilate, tectate. Sexine and nexine approximately of the same thickness. Grain size: $169.2-184.0$ x 140.0$166.3 \mu \mathrm{m}, \mathrm{n}=10$.

\section{Neotatea neblinae Maguire (Plate 2: 37-40) (NY 2415; B. Maguire 42178; Neblina)}

Monads. Isopolar, prolate-spheroidal $(\mathrm{P} / \mathrm{E}=1.04)$, amb triangular, angulaperturate. Tricolporate, zonoaperturate. Ectocolpi long, fusiform, sharp ends $(47.8 \times 4.3 \mu \mathrm{m})$. Ora lalongate $(15.1 \times 28.3 \mu \mathrm{m})$, slightly constricted in the middle, fastigium present. Exine $3.3 \mu \mathrm{m}$ thick, semitectate, perforate. Sexine slightly thicker than nexine. Grain size: 54.2$67.2 \times 53.3-65.3 \mu \mathrm{m}, \mathrm{n}=10$.

\section{Tovomita weddelliana Planch. \& Triana (Plate 2: 41-43)}

(NY 3528; L. Aristeguieta \& G. Agostini 6344; Venezuela)

Monads. Isopolar, prolate $(\mathrm{P} / \mathrm{E}=1.47), \mathrm{amb}$ circular lobate, fossaperturate. Tricolporate, zonoaperturate. Ectocolpi long $(28.0 \mu \mathrm{m})$, marginate, constricted in the equatorial region, sharp ends. Ora only visible in optical section. Exine $1.7 \mu \mathrm{m}$ thick, semitectate, perforate. Sexine and nexine approximately of the same thickness. Grain size: 29.9-37.1 x 20.5-25.6 $\mu \mathrm{m}, \mathrm{n}=10$.

\section{Droseraceae}

\section{Drosera roraimae (Klotzsch ex Diels) Maguire} (Plate 4: 89-91)

(NY 2781; B. Maguire 42338)

Tetrads, uniplanar (rhomboidal and tetragonal) or multiplanar (decussate and tetrahedral). Individual grains inaperturate, heteropolar (distal pole clavatemicroechinate and proximal pole conspicuously folded), oblate-suboblate $(\mathrm{P} / \mathrm{E}=0.75)$, amb subcircular. Exine $3.5 \mu \mathrm{m}$ thick, intectate, microechinate and clavate. Sexine thicker than nexine. Individual grain size: $33.0-44.3 \times 46.6-54.2 \mu \mathrm{m}, \mathrm{n}=16$. Tetrad diameter: 60.3-74.4 $\mu \mathrm{m}, \mathrm{n}=8$.

Comments: Drosera sp. described in SalgadoLabouriau \& Villar (1992) and Rull (2003).

\section{Ericaceae}

\section{Bejaria glauca Humb. \& Bonpl. var. coarctata Mansf. \& Sleumer (Plate 4: 92-94) (NY 1391; B. Maguire \& C. K. Maguire \# 44345; Ecuador)}

Tetrads, multiplanar, tetrahedral or decussate, outline circular slightly lobate. Viscin threads absent. Individual grains heteropolar, oblate $(\mathrm{P} / \mathrm{E}=0.74)$, amb circular, tricolporate. Half ectocolpi $8.1 \mu \mathrm{m}$ long, narrow, slightly marginate. Ora difficult to distinguish. Exine $2.3 \mu \mathrm{m}$ thick, psilate, tectate. Sexine and nexine approximately of the same thickness. Individual grain size: $30.5-37.3 \times 41.1-48.8 \mu \mathrm{m}$, $\mathrm{n}=10$. Tetrad diameter: $56.0-67.3 \mu \mathrm{m}, \mathrm{n}=10$.

Comments: Bejaria aestuans Mutis ex L., in Steyermark et al. (1995-2005).

\section{Cavendishia duidae A. C. Sm. in Gleason (Plate 5: 104-105) \\ (NY 1399; Maguire et al. 29707; Venezuela)}

Tetrads, multiplanar tetrahedral or decussate, outline circular-triangular slightly lobate. Viscin threads absent. Individual grains heteropolar, oblate $(\mathrm{P} / \mathrm{E}=0.72)$, amb circular, tricolporate. Half ectocolpi

\footnotetext{
Plate 3. 47-50, Chorisepalum ovatum var. ovatum (Gentianaceae); 51-53, Macrocarpaea neblinae (Gentianaceae); $54-$ 55, Psittacanthus obovatus (Loranthaceae); 56-58, Diacidia galphimioides (Malpighiaceae); 59-60, Myrcia bolivarenses (Myrtaceae); 61-62, Ouratea gillyana (Ochnaceae); 63-64, Freziera sericea (Ternstroemiaceae); 65-66, Philacra duidae (Ochnaceae); 67-69, Philacra steyermarkii (Ochnaceae); 70, Poecilandra pumila (Ochnaceae); 71, Tyleria spathulata (Ochnaceae); 72, Schoepfia flexuosa (Olacaceae); 73-74, Duranta mutisii (Verbenaceae); 75-76, Banisteriopsis inebrians (Malpighiaceae); 77-79, Borreria laevis (Rubiaceae); 80-81, Heliamphora neblinae var. neblinae (Sarraceniaceae); 82-84, Moronobea jenmanii var. fanshawei (Clusiaceae).
} 

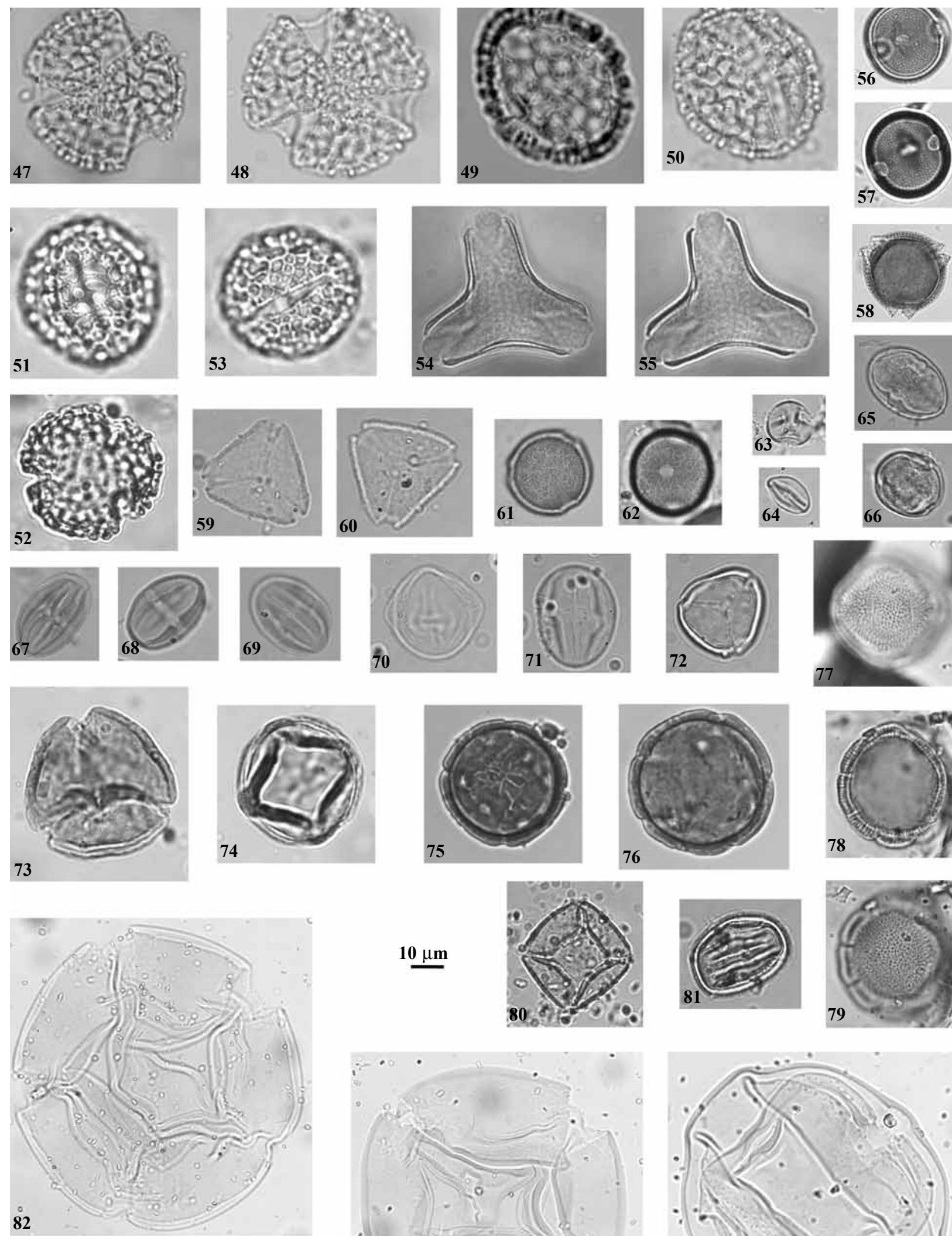

$\mathbf{2 0} \mu \mathbf{m}$
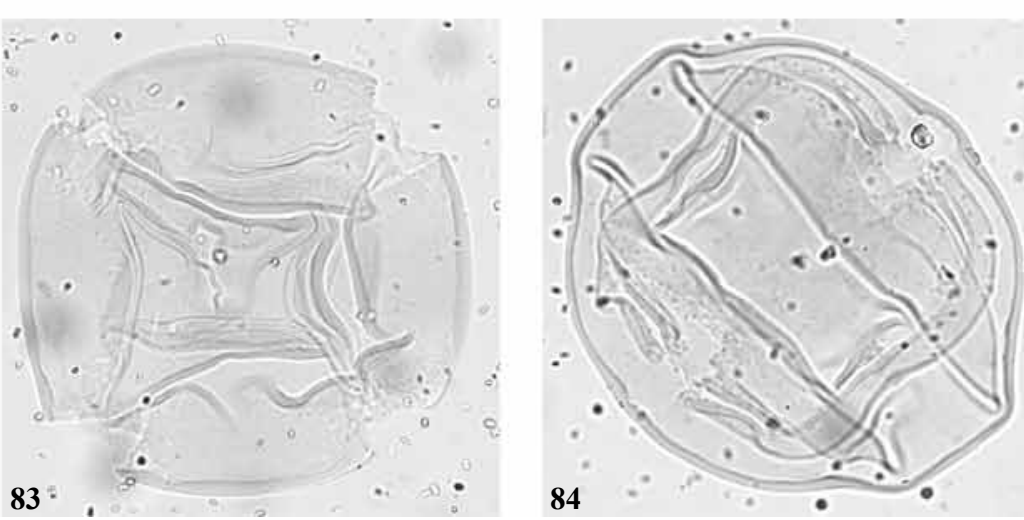
$9.5 \mu \mathrm{m}$ long, narrow, slightly marginate. Ora difficult to distinguish. Exine $1.6 \mu \mathrm{m}$ thick, psilate, tectate. Sexine and nexine indistinguishable for measuring. Individual grain size: $32.2-38.2 \times 40.8-55.4 \mu \mathrm{m}$, $\mathrm{n}=10$. Tetrad diameter: $57.8-71.8 \mu \mathrm{m}, \mathrm{n}=10$.

Disterigma humboldtii Nied. (Plate 5: 108-110)

(NY 1406; J. A. Steyermark, J. J. Wurdack 1156; Venezuela)

Tetrads, multiplanar, tetrahedral or decussate, outline circular-triangular. Viscin threads absent. Individual grains heteropolar, suboblate $(\mathrm{P} / \mathrm{E}=0.80)$, amb circular, tricolporate. Half ectocolpi $17.4 \mu \mathrm{m}$ long, narrow, marginate. Ora difficult to distinguish. Exine $2.7 \mu \mathrm{m}$ thick, psilate, tectate. Sexine and nexine indistinguishable for measuring. Individual grain size: $31.1-35.6 \times 37.4-47.5 \mu \mathrm{m}, \mathrm{n}=19$. Tetrad diameter: $51.5-61.4 \mu \mathrm{m}, \mathrm{n}=19$.

\section{Ledothamnus atroadenus Maguire, Steyerm. \&} Luteyn var. atroadenus (Plate 5: 111-112) (NY 1413; J. J. Wurdack \# 34190; Venezuela)

Tetrads, multiplanar, tetrahedral or decussate, outline circular slightly lobate (sometimes triangularcircular). Viscin threads absent. Individual grains heteropolar, oblate $(\mathrm{P} / \mathrm{E}=0.73)$, amb circular, tricolporate. Half ectocolpi $10.8 \mu \mathrm{m}$ long, narrow, marginate. Ora difficult to distinguish. Exine $2.8 \mu \mathrm{m}$ thick, psilate, tectate. Sexine and nexine approximately of the same thickness. Individual grain size: 28.8-34.6 x 40.2-45.8 $\mu \mathrm{m}, \mathrm{n}=11$. Tetrad diameter: 51.3-60.7 $\mu \mathrm{m}, \mathrm{n}=11$.

Comments: Ledothamnus luteus Maguire, Steyerm. \& Luteyn described in Salgado-Labouriau \& Villar (1992) and Rull (2003).

\section{Mycerinus chimantensis Maguire, Steyerm. \& Luteyn (Plate 5: 106-107) \\ (NY 1422; J. A. Steyermark \& J. J. Wurdack 825; Venezuela)}

Tetrads, multiplanar, tetrahedral or decussate, outline circular slightly lobate. Viscin threads absent. Individual grains heteropolar, suboblate $(\mathrm{P} / \mathrm{E}=0.84)$, amb circular, tricolporate. Half ectocolpi $16.8 \mu \mathrm{m}$ long but often difficult to distinguish. Ora difficult to distinguish. Exine $2.2 \mu \mathrm{m}$ thick, psilate, tectate. Sex- ine and nexine approximately of the same thickness. Individual grain size: $39.3-48.4 \times 43.6-53.6 \mu \mathrm{m}, \mathrm{n}=$ 10. Tetrad diameter: 71.6-80.5 $\mu \mathrm{m}, \mathrm{n}=7$.

\section{Notopora schomburgkii Hook.f. (Plate 6: 115-116) (NY 1668; B. Maguire et al. 46150A; British Guiana)}

Tetrads, multiplanar, tetrahedral or decussate, outline triangular or quadrangular. Viscin threads absent. Individual grains heteropolar, oblate $(\mathrm{P} / \mathrm{E}=0.74)$, amb circular, tricolporate. Half ectocolpi $20.3 \mu \mathrm{m}$ long, narrow, marginate. Ora difficult to distinguish. Exine $3.1 \mu \mathrm{m}$ thick, psilate, tectate. Sexine and nexine approximately of the same thickness. Individual grain size: $40.5-49.9 \times 56.5-63.7 \mu \mathrm{m}, \mathrm{n}=10$. Tetrad diameter: $75.7-90.4 \mu \mathrm{m}, \mathrm{n}=10$.

\section{Notopora smithiana Steyerm. \& Maguire (Plate} 6: 117-120)

\section{(NY 3494; J. A. Steyermark \& J. J. Wurdack} 823; Chimantá, Venezuela)

Tetrads, multiplanar, tetrahedral or decussate, outline circular-triangular. Viscin threads absent. Individual grains heteropolar, oblate $(\mathrm{P} / \mathrm{E}=0.72)$, amb circular, tricolporate. Half ectocolpi $19.1 \mu \mathrm{m}$ long, $3.8 \mu \mathrm{m}$ wide, sharp ends, sometimes appear narrow. Ora difficult to distinguish, probably lalongate $(10.4 \mu \mathrm{m}$ wide). Exine $2.9 \mu \mathrm{m}$ thick, microrugulate, tectate. Sexine and nexine approximately of the same thickness. Individual grain size: 37.3-42.1 x 52.5-59.0 $\mu \mathrm{m}, \mathrm{n}=11$. Tetrad diameter: 70.1-77.3 $\mu \mathrm{m}, \mathrm{n}=11$.

\section{Orthaea paruensis Spruce (Plate 6: 121-124) (NY 1424; K. D. Phelps \& C. B. Hitchcock 477; Venezuela)}

Tetrads, multiplanar, tetrahedral or decussate, outline circular-triangular. Viscin threads absent. Individual grains heteropolar, suboblate $(\mathrm{P} / \mathrm{E}=0.77)$, amb circular, tricolporate. Half ectocolpi $13.6 \mu \mathrm{m}$ long, narrow, marginate (half margo: $16.2 \times 3.3 \mu \mathrm{m}$ ). Ora difficult to distinguish but they seem lalongate (10.7 $\mu \mathrm{m}$ wide). Exine $2.7 \mu \mathrm{m}$ thick, psilate, tectate. Sexine and nexine approximately of the same thickness. Individual grain size: $30.1-34.7 \times 39.1-45.7 \mu \mathrm{m}$, $\mathrm{n}=12$. Tetrad diameter: 55.9-62.1 $\mu \mathrm{m}, \mathrm{n}=12$. 

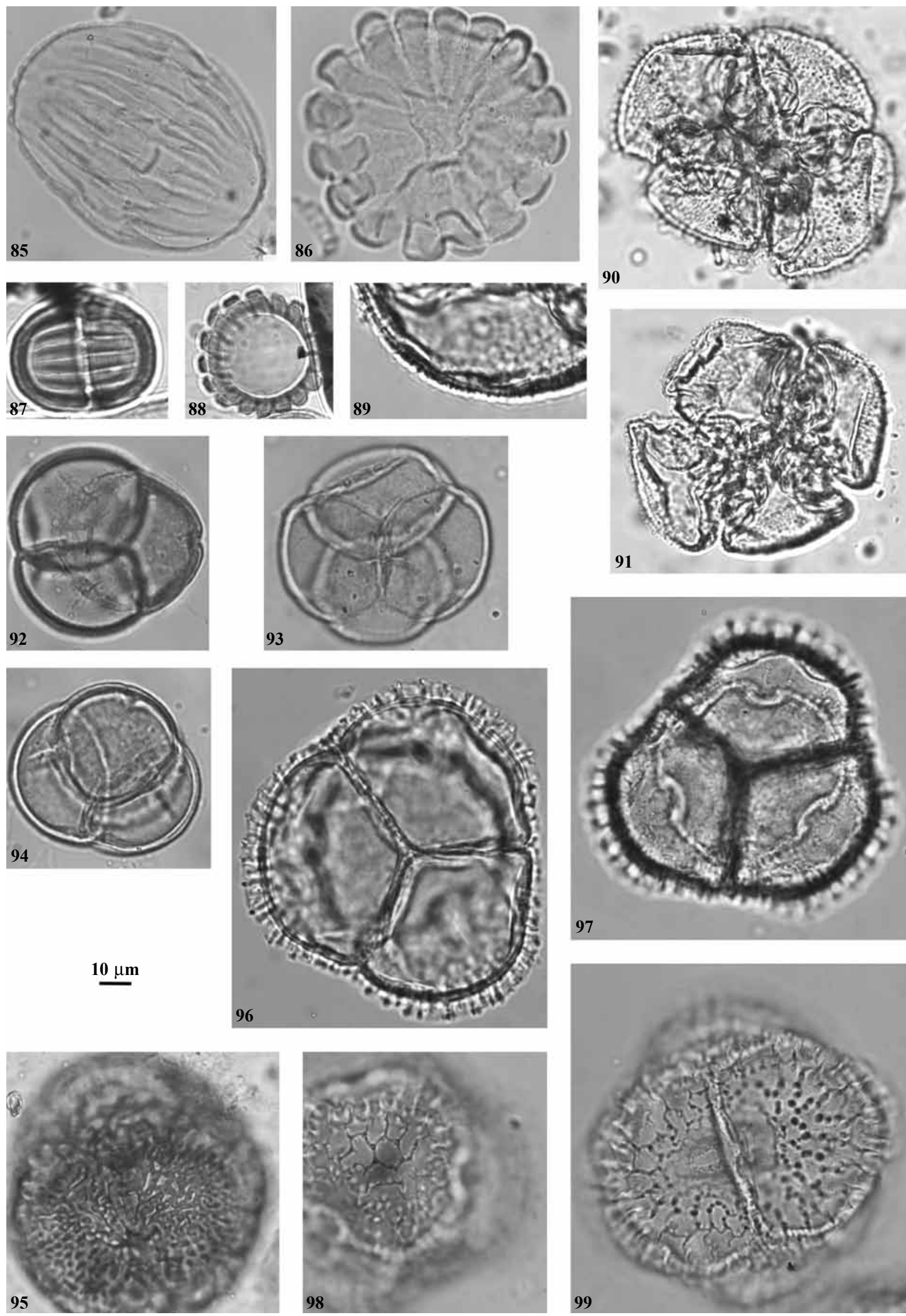
Tepuia cardonae A. C. Sm. (Plate 5: 113-114) (NY 1671; J. A. Steyermark \& J. J. Wurdack 1039; Venezuela)

Tetrads, multiplanar, tetrahedral or decussate, outline circular or subtriangular. Viscin threads absent. Individual grains heteropolar, oblate $(\mathrm{P} / \mathrm{E}=0.69)$, amb circular, tricolporate. Half ectocolpi $10.8 \mu \mathrm{m}$ long, narrow. Ora difficult to distinguish. Exine $3.2 \mu \mathrm{m}$ thick, psilate, tectate. Sexine and nexine approximately of the same thickness. Individual grain size: $24.1-39.1 \times 38.5-54.9 \mu \mathrm{m}, \mathrm{n}=9$. Tetrad diameter: 47.6-62.6 $\mu \mathrm{m}, \mathrm{n}=9$.

Comments: Tepuia venusta Camp. described in Salgado-Labouriau \& Villar (1992) and Rull (2003).

Thibaudia cupatensis Huber (Plate 6: 125-126) (NY 1436; B. Maguire et al. \# 42263; Venezuela)

Tetrads, multiplanar, tetrahedral or decussate, outline circular, slightly lobate. Viscin threads absent. Individual grains heteropolar, suboblate $(\mathrm{P} / \mathrm{E}=0.76)$, amb circular, tricolporate. Half ectocolpi $16.8 \mu \mathrm{m}$ long, narrow, marginate. Ora difficult to distinguish. Exine $2.4 \mu \mathrm{m}$ thick, psilate, tectate. Sexine and nexine approximately of the same thickness. Individual grain size: $39.4-46.9 \times 52.3-58.6 \mu \mathrm{m}, \mathrm{n}=10$. Tetrad diameter: 68.2-78.8 $\mu \mathrm{m}, \mathrm{n}=10$.

Comments: Thibaudia nutans Klotzsch ex Mansf. described in Salgado-Labouriau \& Villar (1992) and Rull (2003).

\section{Gentianaceae}

\section{Chorisepalum ovatum Gleason var. ovatum (Plate 3: 47-50)}

(NY 2500; B. Maguire et al. 27921; Venezuela)

Monads. Isopolar, subprolate $(\mathrm{P} / \mathrm{E}=1.21), \mathrm{amb}$ circular. Tricolporate, zonoaperturate. Ectocolpi long and wide $(36.1 \times 3.9 \mu \mathrm{m})$, sharp ends, constricted at the equatorial region. Ora apparently lolongate, but difficult to distinguish. Exine 4.5 $\mu \mathrm{m}$ thick, semitectate, reticulate, irregularly heterobrochate (lumina smaller in polar regions), simplicolumellate. Sexine thicker than nexine. Grain size: $48 \cdot 0-54.2 \times 35 \cdot 0-44.9 \mu \mathrm{m}, \mathrm{n}=6$.

\section{Irlbachia cardonae (Gleason) Maguire (Plate 6:} 127-129)

\section{(NY 2531; B. Maguire et al. 33917; Venezuela)}

Polyad, constituted by 16 grains, outline circular. Individual grains heteropolar (only distal pole echinate), suboblate $(\mathrm{P} / \mathrm{E}=0.78)$, amb circular. Apertures difficult to distinguish due to the sculpture (Gentianaceae are normally tricolporate). Exine $4.7 \mu \mathrm{m}$ thick (excluding spines), reticulate, semitectate, echinate (4-5 big curved spines per grain with rounded ends: $22.5 \mu \mathrm{m}$ long, $13.4 \mu \mathrm{m}$ base diameter), supratectal granula and verrucae irregularly distributed. Sexine thicker than nexine. Individual grain size: 43.0-57.8 x 56.9-69.7 $\mu \mathrm{m}, \mathrm{n}=7$. Polyad diameter 190.9-210.2 $\mu \mathrm{m}$ (excluding spines), $\mathrm{n}=5$.

\section{Macrocarpaea neblinae Maguire \& Steyerm. (Plate 3: 51-53) \\ NY 2723; B. Maguire et al. 37103; Venezuela)}

Monads. Isopolar, prolate-spheroidal $(\mathrm{P} / \mathrm{E}=1.06)$, amb circular. Tricolporate, zonoaperturate. Ectocolpi long, sharp ends, sometimes slightly rounded, fusiform $(28.5 \times 2.3 \mu \mathrm{m})$, marginate. Ora elliptic $(6.5 \times 4.3 \mu \mathrm{m})$, lolongate. Exine $3.8 \mu \mathrm{m}$ thick, semitectate, reticulate irregularly heterobrochate, simpliand duplicolumellate. Sexine thicker than nexine. Grain size: 41.7-45.1 x 38.8-43.0 $\mu \mathrm{m}, \mathrm{n}=10$.

Comments: Macrocarpaea tepuiensis (Gleason) Steyermark described in Rull (2003).

Neblinantha neblinae Maguire (Plate 5: 100-103) (NY 2541; B. Maguire et al. 60527; Neblina)

Tetrads, multiplanar, tetrahedral or decussate. Individual grains heteropolar, oblate $(\mathrm{P} / \mathrm{E}=0.72), \mathrm{amb}$ circular-slightly lobate. Tricolporate, colpi and ora difficult to observe due to the sculpture pattern. Exine $7.7 \mathrm{~mm}$ thick, semitectate, reticulate, heterobrochate, simplicolumellate. Sexine thicker than nexine. Individual grain size: $47.5-54.0 \times 63.2-77.9 \mu \mathrm{m}, \mathrm{n}=10$. Tetrad diameter: $90.3-103.8 \mu \mathrm{m}, \mathrm{n}=10$.

\section{Schultesia heterophylla Miq. (Plate 4: 96-99)} (NY 2565; J. J. Wurdack \& N. G. L. Guppy 15; Venezuela)

Tetrads, multiplanar, tetrahedral or decussate, outline circular-triangular. Individual grains hetero-

Plate 5. 100-103, Neblinantha neblinae (Gentianaceae); 104-105, Cavendishia duidae (Ericaceae); 106-107, Mycerinus chimantensis (Ericaceae); 108-110, Disterigma humboldtii (Ericaceae); 111-112, Ledothamnus atroadenus var. atroadenus (Ericaceae); 113-114, Tepuia cardonae (Ericaceae). 

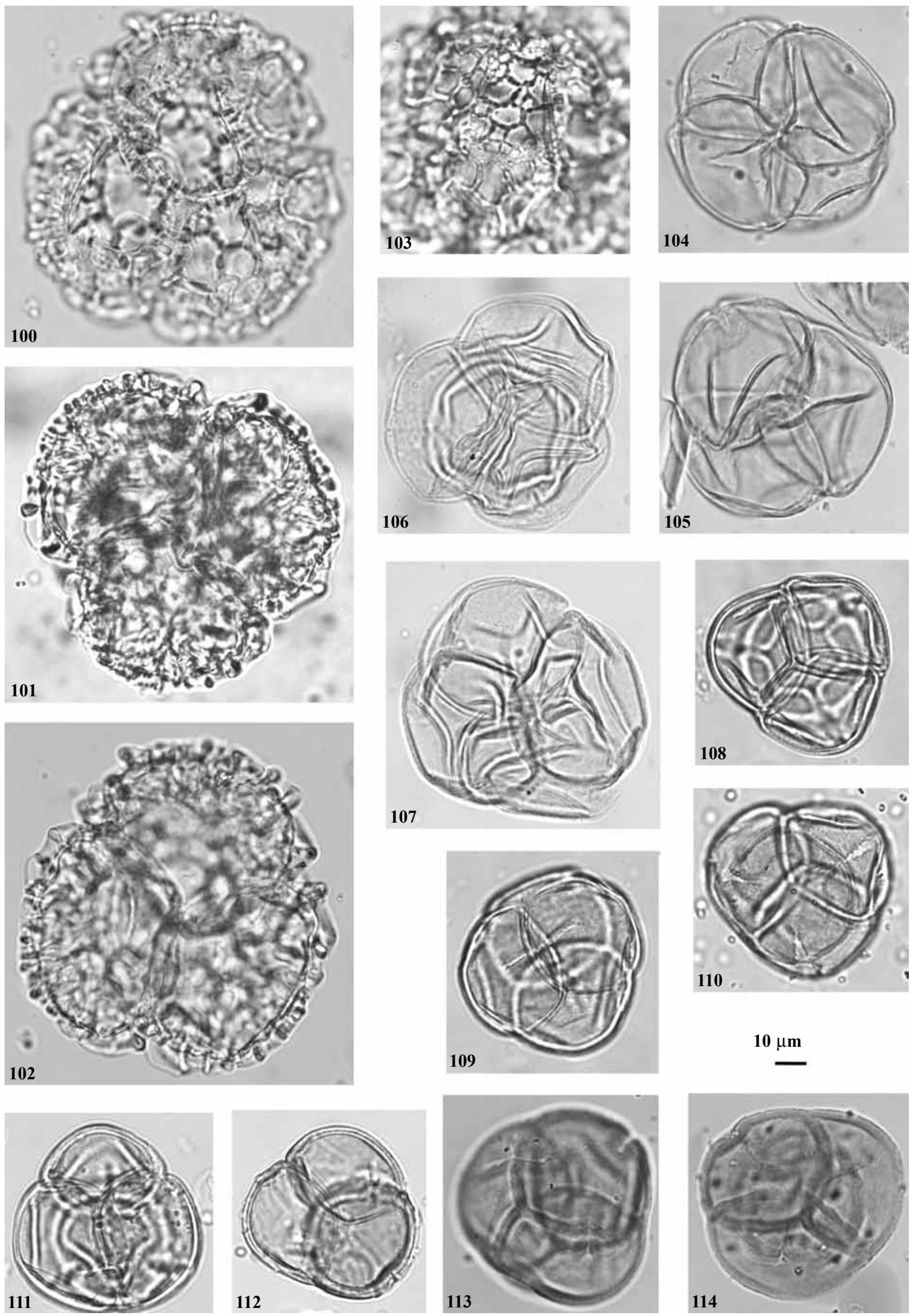
polar, oblate to suboblate $(\mathrm{P} / \mathrm{E}=0.75)$, amb circular. Triporate, pori elliptic (7.2 $\mu \mathrm{m}$ long, $8.9 \mu \mathrm{m}$ wide), annulate. Exine $8.0 \mu \mathrm{m}$ thick, semitectate, reticulate heterobrochate. Sexine thicker than nexine. Individual grain size: $46.7-54.0 \times 63.4-73.3 \mu \mathrm{m}, \mathrm{n}=11$. Tetrad diameter: $88.3-97.9 \mu \mathrm{m}, \mathrm{n}=11$.

\section{Symbolanthus elisabethae Gilg (Plate 4: 95) (NY 1266; J. A. Steyermark 75662; Venezuela, Bolivar, June 1953)}

Tetrads, multiplanar, tetrahedral or decussate. Apertures difficult to observe due to the sculptural pattern. Exine 5-6 $\mu \mathrm{m}$ thick, semitectate, reticulate heterobrochate, simplicolumellate. Sexine thicker than nexine. Tetrad diameter: 85.8-90.0 $\mu \mathrm{m}, \mathrm{n}=4$.

\section{Tapeinostemon longiflorum Maguire \& Steyerm. (Plate 2: 44-46)}

(NY 2579; J. A. Steyermark 103937; Venezuela)

Monads. Isopolar, subprolate $(\mathrm{P} / \mathrm{E}=1.30), \mathrm{amb}$ circular. Tricolporate, zonoaperturate. Ectocolpi long $(30.3 \mu \mathrm{m})$ and narrow, sharp ends. Ora circular (6.4 $\mu \mathrm{m}$ diameter). Exine $3.0 \mu \mathrm{m}$ thick, semitectate, perforate. Sexine thicker than nexine. Grain size: $43.3-53.9 \times 35.6-42.0 \mu \mathrm{m}, \mathrm{n}=6$.

\section{Lentibulariaceae}

\section{Utricularia foliosa L. (Plate 4: 85-86)}

\section{(NY 1646; E. Asplund 5793; Ecuador)}

Monads. Isopolar, subprolate $(\mathrm{P} / \mathrm{E}=1.29), \mathrm{amb}$ circular slightly multilobate. Polyzonocolporate (17 apertures). Ectocolpi long $(68.7 \mu \mathrm{m})$ and narrow, endocingulum present (zonorate). Exine $2.0 \mu \mathrm{m}$ thick, psilate. Sexine and nexine indistinguishable. Grain size: $81.63-88.1 \times 63.9-66.5 \mu \mathrm{m}, \mathrm{n}=3$.

Comments: Utricularia quelchii N. E. Brown described in Rull (2003) and Salgado-Labouriau \& Villar (1992).

\section{Loranthaceae}

Psittacanthus obovatus Eichl. (Plate 3: 54-55)

(NY 1612; P. C. Hutchinson 1363 H. G. H.; Peru) Monads. Amb subtriangular concave, angulaper- turate. Tricolp(or)ate, zonoaperturate, syncolp(or) ate. Exine psilate, tectate.

Comments: One single pollen grain found (PV).

\section{Malpighiaceae}

Banisteriopsis inebrians C. V. Morton (Plate 3: 75-76)

(NY 2703; B. A. Krukoff-8550; Brazil)

Monads. Spheroidal. Pantoporate, with irregular colpoids in several directions. Pori elliptic $(3.4 \times 2.1 \mu \mathrm{m})$, with diffuse outline. Exine $3.8 \mu \mathrm{m}$ thick, tectate, psilate. Sexine thicker than nexine. Grain diameter: 37.5-46.2 $\mu \mathrm{m}, \mathrm{n}=10$.

Diacidia galphimioides Griseb (Plate 3: 56-58) (NY 670; B. Maguire 44091)

Monads. Isopolar, prolate-spheroidal $(\mathrm{P} / \mathrm{E}=1.04)$, amb circular-triangular, angulaperturate. Tricolporate, zonoaperturate. Ectocolpi short, sharp ends, fusiform $(13.2 \times 3.2 \mu \mathrm{m})$. Ora elliptic, lalongate $(4.4 \times 6.2 \mu \mathrm{m})$, fastigium present. Exine $1.7 \mu \mathrm{m}$ thick, semitectate, reticulate heterobrochate, lumina size decreasing towards the apertures and the polar region. Nexine slightly thicker than sexine. Grain size: $24.7-28.9 \times 22.9-27.4 \mu \mathrm{m}, \mathrm{n}=11$.

Comments: The slide has the epithet duckei, however this name was never published.

\section{Myrtaceae}

Myrcia bolivarensis (Steyerm.) McVaugh (Plate 3: 59-60)

(NY 2193; S. S. Tillett \& C. L. Tillett 45012; B. Guiana)

Monads. Isopolar, amb triangular, angulaperturate. Tricolporate, syncolporate. Exine $1.7 \mu \mathrm{m}$ thick, reticulate or areolate with ondulating surface (difficult to observe due to bad preservation). Sexine and nexine difficult to distinguish for measuring. Grain size: $29.2-33.8 \mu \mathrm{m}$, median in $\mathrm{PV}, \mathrm{n}=10$.

Comments: Description based on grains in PV. Myrcia bonnetia-sylvestris (Steyerm.) Steyermark described in Salgado-Labouriau \& Villar (1992) and Rull (2003). 

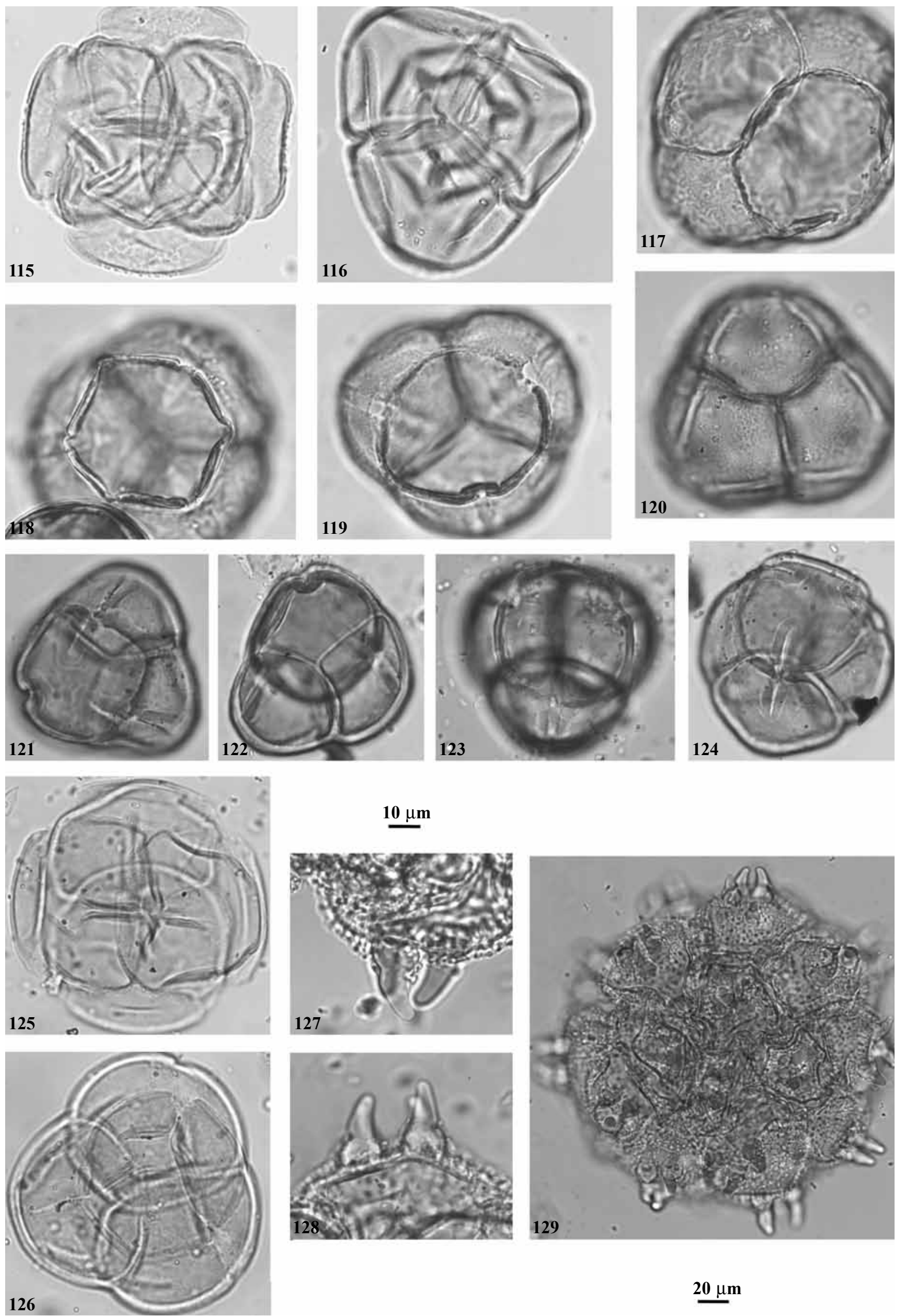


\section{Ochnaceae}

\section{Ouratea gillyana (Dwyer) Sandwith \& Maguire} (Plate 3: 61-62)

(B. Maguire \& D. B. Fanshawe 32629; 311)

Monads. Isopolar, spheroidal $(\mathrm{P} / \mathrm{E}=1.00)$, amb circular. Tricolporate, zonoaperturate. Ectocolpi apparently fusiform but difficult to observe (9.9 $\mu \mathrm{m}$ long, $\mathrm{n}=2$ ), ora slightly elliptic lolongate $(4.7 \times 3.9 \mu \mathrm{m})$. Exine $1.4 \mu \mathrm{m}$ thick, psilate and tectate (columellae visible through the tectum). Sexine and nexine approximately of the same thickness. Grain size: $23.2-26.7 \times 23.1-27.1 \mu \mathrm{m}, \mathrm{n}=10$.

\section{Philacra duidae (Gleason) Dwyer (Plate 3: 65-66)}

\section{(NY 279; G. H. H. Tate 529)}

Monads. Isopolar, prolate $(\mathrm{P} / \mathrm{E}=1.36)$, amb circular. Tricolporate, zonoaperturate. Ectocolpi long $(19.5 \mu \mathrm{m})$ and narrow. Ora elliptic, lalongate: $3.5 \mu \mathrm{m}$ long $(\mathrm{n}=$ 7) $x 7.9 \mu \mathrm{m}$ wide $(\mathrm{n}=4)$. Exine $1.0 \mu \mathrm{m}$ thick, psilate and tectate (columellae visible through the tectum). Sexine and nexine indistinguishable for measuring. Grain size: $24.2-26.9 \times 17.9-19.3 \mu \mathrm{m}, \mathrm{n}=10$.

Philacra steyermarkii Maguire (Plate 3: 67-69) (NY 277; J. A. Steyermark 93615)

Monads. Isopolar, prolate $(\mathrm{P} / \mathrm{E}=1.40), \mathrm{amb}$ apparently circular-triangular, angulaperturate. Tricolporate, zonoaperturate. Ectocolpi long $(21.9 \mu \mathrm{m})$ and narrow, constricted in the equatorial region. Ora elliptic, lalongate $(3.4 \times 7.9 \mu \mathrm{m})$, sometimes constricted. Exine $1.6 \mu \mathrm{m}$ thick, psilate and tectate (columellae visible through the tectum). Sexine and nexine approximately of the same thickness. Grain size: $26.2-31.1 \times 18.6-22.1 \mu \mathrm{m}, \mathrm{n}=12$.

\section{Poecilandra pumila Steyerm. (Plate 3: 70)}

(NY 297; B. Maguire \& S. S. Tillett 43800)

Monads. Isopolar, oblate-spheroidal $(\mathrm{P} / \mathrm{E}=0.99)$, amb circular. Tricolporate, zonoaperturate. Ectocolpi long $(21.2 \mu \mathrm{m})$ and narrow, ora apparently elliptic lalongate, fastigium present. Exine $1.3 \mu \mathrm{m}$ thick, psilate and tectate. Sexine and nexine indistinguishable for measuring. Grain size: 27.3-32.2 x 26.6-31.7 $\mu \mathrm{m}, \mathrm{n}=10$.

\section{Tyleria spathulata Gleason (Plate 3: 71) \\ (NY 295; B. Maguire et al. 30092)}

Monads. Isopolar, prolate-spheroidal $(\mathrm{P} / \mathrm{E}=1.13)$, amb circular slightly lobate. Tricolporate, zonoaperturate. Ectocolpi long $(23.4 \mu \mathrm{m}, \mathrm{n}=3)$ and narrow (2.0 $\mu \mathrm{m}, \mathrm{n}=1)$, sharp ends, slightly marginate. Ora elliptic, lalongate $(3.2 \times 6.4, \mathrm{n}=1)$. Exine $1.3 \mu \mathrm{m}$ thick, psilate, tectate. Sexine and nexine approximately of the same thickness. Grain size: $24.0-32.5 \times 20.2-26.4 \mu \mathrm{m}, \mathrm{n}=11$.

\section{Olacaceae}

Schoepfia flexuosa Roem. \& Schult. (Plate 3: 72) (NY 3037; J. A. Steyermark \& J. J. Wurdack 326, Feb. 2, 1955; Chimantá, Venezuela)

Monads. Isopolar, amb triangular-rounded, angulaperturate. Tricolporate, syncolporate. Exine $1.52 \mu \mathrm{m}$ thick (measured in PV), psilate. Sexine and nexine approximately of the same thickness. Grain size: $25.7-32.1 \mu \mathrm{m}$, median in PV, $\mathrm{n}=6$.

Comments: Described in Maguire et al. (1974), on the same slide.

\section{Polygalaceae}

Monnina chanduyensis Chod. (Plate 4: 87-88) (NY 809; F. W. Pennell 14467; Peru)

Monads. Isopolar, prolate $(\mathrm{P} / \mathrm{E}=1.34), \mathrm{amb}$ circular slightly multilobate. Polyzonocolporate (17 colpori). Ectocolpi long $(34.4 \mu \mathrm{m})$ and narrow, endocingulum present (zonorate). Exine $4.0 \mu \mathrm{m}$ thick, psilate to slightly scabrate, tectate (columellae visible through the tectum). Nexine thicker than sexine. Grain size: 45.7-52.7 x 30.8-39.5 $\mu \mathrm{m}, \mathrm{n}=6$.

\section{Rapateaceae}

Kunhardtia rhodantha Maguire (Plate 1: 1-3) (NY 3056; B. Maguire \& L. Politi 27954; Cerro Sipapo, Venezuela, Jan. 1, 1949)

Monads. Heteropolar, oblate $(\mathrm{P} / \mathrm{E}=0.65)$. Monosulcate, sulcus long $(39.8 \mu \mathrm{m})$. Exine $1.5 \mu \mathrm{m}$ thick, psilate, tectate. Sexine and nexine approximately of the same thickness. Grain size: 27.4-36.9 x 33.0-56.6 $\mu \mathrm{m}, \mathrm{n}=10$.

Comments: Described in Carlquist (1961).

\section{Rubiaceae}

Borreria laevis (Lam.) Griseb. (Plate 3: 77-79) (NY 815; H. H. Smith 87; Colombia)

Monads. Isopolar, oblate-spheroidal $(\mathrm{P} / \mathrm{E}=0.94)$, amb circular. Polyzonocolpate ( 9 colpi), short and narrow (5.4 $\mu \mathrm{m}$ long, $0.8 \mu \mathrm{m}$ wide), ends sharp. 
Exine $2.7 \mu \mathrm{m}$ thick, tectate, psilate (columellae visible through the tectum). Sexine thicker than nexine. Grain size: $29.8-36.6 \times 30.8-38.5 \mu \mathrm{m}, \mathrm{n}=8$.

Comments: Borreria remota (Lam.) Bacigalupo \& E. L. Cabral, in Steyermark et al. (1995-2005).

\section{Sarraceniaceae}

\section{Heliamphora neblinae Maguire var. neblinae} (Plate 3: 80-81)

\section{(NY 2736; B. Maguire et al. 37019; Venezuela)}

Monads. Isopolar, prolate $(\mathrm{P} / \mathrm{E}=1.41), \mathrm{amb}$ usually quadrangular, angulaperturate. Tretracolporate, zonoaperturate. Ectocolpi long $(20.3 \mu \mathrm{m})$ and narrow. Os indistinct, sometimes visible in optical section. Exine $1.3 \mu \mathrm{m}$ thick, psilate, tectate. Sexine and nexine approximately of the same thickness. Grain size: $30.0-31.2 \times 21.4-22.8 \mu \mathrm{m}, \mathrm{n}=3$.

Comments: Heliamphora minor Gleason described in Salgado-Labouriau \& Villar (1992) and Rull (2003).

\section{Ternstroemiaceae}

\section{Freziera sericea Humb. \& Bonpl. (Plate 3: 63-64) (NY 209; 892)}

Monads. Isopolar, prolate $(\mathrm{P} / \mathrm{E}=1.46)$, amb circular. Tricolporate, zonoaperturate. Ectocolpi long $(11.7 \mu \mathrm{m})$ and narrow, os indistinct. Exine $0.7 \mu \mathrm{m}$ thick, psilate. Sexine and nexine indistinguishable for measuring. Grain size: $14.0-15.5 \times$ 9.3-11.2 $\mu \mathrm{m}, \mathrm{n}=10$.

Comments: Eurya sericea (Kunth) Szyszyl., in Hokche et al. (2008).

\section{Velloziaceae}

Barbacenia magalhaesii L. B. Sm. (Plate 1:4-5) (NY 438; B. Maguire et al. 49062; Brazil)

Monads. Heteropolar, oblate $(\mathrm{P} / \mathrm{E}=0.61)$. Monosulcate, sulcus long. Exine $<1 \mu \mathrm{m}$ thick, perforate. Sexine and nexine indistinguishable for measuring. Grain size: $16.9-24.6 \times 30.6-36.4 \mu \mathrm{m}, \mathrm{n}=5$.

\section{Verbenaceae}

Duranta mutisii L. f. (Plate 3: 73-74) (NY 1526; W. H. Camp E-5203, Ecuador)

Monads. Isopolar, spheroidal $(\mathrm{P} / \mathrm{E}=1.00), \mathrm{amb}$ triangular, angulaperturate. Tricolporate, zonoaper- turate. Ectocolpi long, sharp ends. Exine $3.8 \mu \mathrm{m}$ thick, psilate, tectate. Sexine thicker than nexine. Grain size: $39.7-42.6$ x 40.7-40.9 $\mu \mathrm{m}, \mathrm{n}=3$.

Comments: Duranta obtusifolia, in Steyermark et al. (1995-2005).

\section{ACKNOWLEDGEMENTS}

The authors are grateful to O. Huber, who noticed the existence of the Maguire pollen collection and encouraged its study. The comments of one anonymous reviewer contributed to the improvement of the manuscript. Funding was provided by the Higher Council for Scientific Research (CSIC) of Spain and the Biological Sciences Commission of the Institute for Catalan Studies (IEC).

\section{REFERENCES}

Berry, P. E. \& Riina, R. 2005. Insights into the diversity of the Pantepui flora and the biogeographic complexity of the Guayana Shield. Biol. Skr. 55: 145-167.

Briceño, H. O. \& Schubert, C. 1990. Geomorphology of the Gran Sabana, Guayana Shield, southeastern Venezuela. Geomorphology 3: 125.141.

Carlquist, S. 1961. Pollen morphology of Rapateaceae. Aliso 5: 39-66.

Funk, V. A., Susanna, A., Stuessy, T. F. \& Bayer, R. J. (Eds.). 2009. Systematics, evolution, and Biogeography of Compositae. IAPT, Vienna.

Gorzula, S. \& Huber, O. 1992. Consideraciones finales. In: Huber, O. (Ed.), El Macizo del Chimantá. Un ensayo ecológico tepuyano. Oscar Todtmann Eds., Caracas: 325-330.

Hokche, O., Berry, P. E. \& Huber, O. (Eds.). 2008. Nuevo Catálogo de la Flora Vascular de Venezuela. Fundación Instituto Botánico de Venezuela, Caracas.

Howard, R. A. \& Boom, B. M. 1990. Bassett Maguire-An annotated biography. Mem. New York Bot. Gard. 64: 1-28.

Huber, O. 1988. Guayana Highlands versus Guayana Lowlands, a reappraisal. Taxon 37 (3): 595-614.

Huber, O. 1994. Recent advances in the phytogeography of the Guayana region, South America. Mém. Soc. Biogéogr. 4: 53-63.

Huber, O. 1995a. Geographical and physical features. In: Berry, P. E., Holst, B. K. \& Yaskievych, K. (Eds.), Flora of the Venezuelan Guayana 1, Introduction. Missouri Botanical Garden Press, St. Louis: 1-61.

Huber, O. 1995b. Conservation of the Venezuelan Guayana. In: Berry, P. E., Holst, B. K. \& Yaskievych, K. (Eds.), Flora of the Venezuelan Guayana 1, Introduction. Missouri Botanical Garden Press, St. Louis: 193-218.

Huber, O. 2005. Diversity of vegetation types in the Guayana region: an overview. Biol. Skr. 55: 169-188.

Maguire, B., Wurdack, J. J. \& Huang, Y. 1974. Pollen grains of some American Olacaceae. Grana 14: 26-38.

Nogué, S., Rull, V. \& Vegas-Vilarrúbia, T. 2009. Modeling biodiversity loss by global warming on Pantepui, northern South America. Clim. Change 94: 77-85. 
Punt, W., Hoen, P. P., Blackmore, S., Nilsson, S. \& Le Thomas, A. 2007. Glossary of pollen and spore terminology. Rev. Palaeobot. Palynol. 143: 1-81. Retrieved Oct 25, 2010, from http://www3.bio.uu.nl/palaeo/glossary

Rull, V. 2003. An illustrated key for the identification of pollen from Pantepui and the Gran Sabana (Eastern Venezuelan Guayana). Palynology 27: 99-133.

Rull, V. \& Vegas-Vilarrúbia, T. 2006. Unexpected biodiversity loss under global warming in the neotropical Guayana Highlands. Global Change Biol. 12: 1-9.

Rull, V. \& Vegas-Vilarrúbia, T. 2008. Biopiracy rules hinder conservation efforts. Nature 453: 26.

Rull, V., Vegas-Vilarrúbia, T., Nogué, S. \& Montoya, E. $2008 a$. Bureaucratic obstruction of conservation science in the Guayana highlands. Conservation Biol. 22: 508-509.

Rull, V., Vegas-Vilarrúbia, T., Nogué, S. \& Huber, O. $2008 b$. Conservation of the unique neotropical vascular flora of the Guayana highlands in the face of global warming. Conservation Biol. 23: 1323-1327.

Rull, V. 2009. Pantepui. In: Gillespie, R. G. \& Clague, D. A. (Eds.), Encyclopedia of Islands. University of California Press, Berkeley: 717-720.

Rull, V. 2010. The Guayana Highlands: a natural laboratory for the biogeographical and evolutionary study of the neotropical flora. In: Sánchez-Villagra, M., Carlini, A. A. \& Aguilera,
O. A. (Eds.), Urumaco and Venezuelan palaeontology-the fossil record of the northern Neotropics. Indiana University Press, Bloomington: 84-102.

Rull, V., Abbott, M. B., Vegas-Vilarrúbia, T., Bezada, M., Montoya, E. Nogué, E. \& González, C. 2010. Paleoenvironmental trends in Venezuela during the last glacial cycle. In: Sánchez-Villagra, M., Carlini, A. A. \& Aguilera, O. A. (Eds.). Urumaco and Venezuelan palaeontology-the fossil record of the northern Neotropics. Indiana University Press, Bloomington: 52-83.

Salgado-Labouriau, M. L. \& Villar, L. 1992. Contribución a la flora polínica de los tepuyes. In: Huber, O. (Ed.), El Macizo del Chimantá. Un ensayo ecológico tepuyano. Oscar Todtmann Eds., Caracas: 219-236.

Steyermark, J. A., Berry, P. E., Yatshievych, K. \& Holst, B. K. (Eds). 1995-2005. Flora of the Venezuelan Guayana. Missouri Botanical Garden, St. Louis \& Timber Press, Portland, OR.

Tellería, M. C. 2008. Taxonomic significance of pollen types in the Guayana Highland-centered composite genera of Mutisioideae (Asteraceae). Bot. J. Linn. Soc. 156: 327-340.

Ubiergo, P., Lapp, M. \& Torrecilla, P. 2009. Morfología del polen de especies de Gongylolepis (Mutisieae: Asteraceae) de la Guayana venezolana. Anales Jard. Bot. Madrid 66: 93-107. 
Appendix. List of species/slides of the Maguire collection analyzed, which pollen grains are unsuitable for a morphological study.

\author{
Acanthaceae \\ Justicia carthaginensis Jacq. (NY 846; O. L. Haught 4753; Colombia)
}

\title{
Annonaceae
}

Duguetia riberensis Aristeg. ex Mass \& Boon (NY 658; L. Aristeguieta 5308; S. America)

\section{Boraginaceae}

Cordia leucophylctis Hook. f. (NY 862; Chapin 1125; Indefatigable Is., Galapagos)

\section{Cyatheaceae}

Cyathea arborea (L.) Sm. (NY 2995; E. L. Little, Jr., 22 June 1950; El Verde, Puerto Rico)

\section{Dennstaedtiaceae}

Hypolepis repens (L.) Presl. (NY 1546; Cuatrecasas 16092; Colombia)

\section{Dryopteridaceae}

Diplazium lindbergii (Mett.) Christ (NY 1524; Holdridge 1608; Ecuador)

\section{Fabaceae}

Inga aff. punctata Willd. (NY 769; G. Tessmann 3443; Peru)

\section{Lamiaceae}

Hyptis capitata Jacq. (NY 754; Toro 44; Colombia)

\section{Myrsinaceae}

Cybianthus brownii Gleason (NYBG 2363; Maguire 32104; Brit Guiana)

\section{Myrtaceae}

Eugenia florida D. C. (NY 2170; H. S. Irwin et al. 55206; Suriname)

\section{Ochnaceae}

Elvasia quinqueloba Spruce (\# 34812; B. Maguire \& J. J. Wurdack, 287) 